\section{EDTTORTAL BOARD}

Lida Anestidou, DVM, PhD

Program Officer, Institute for Laboratory Animal Medicine,

The National Academies, Washington, DC.

Kathryn Bayne, MS, PhD, DVM, DACLAM, CAAB

Senior Director and Director of Pacific Rim Activities,

AAALAC International, Waikoloa, HI.

Joseph T. Bielitzki, MS, DVM

Associate Director, Nanoscience Center,

Associate Director, Nanoscience Center,
University of Central Florida, Orlando, FL.

Cyndi Brown, DVM, DABVP (Avian Practice)
Red Bank Veterinary Hospital, Tinton Falls, NJ.

Joseph W. Carraway, DVM, MS

Director of Toxicology, NAMSA, Northwood, $\mathrm{OH}$.

Thomas M. Donnelly, DVM, DACLAM

The Kenneth S. Warren Institute, Ossining, NY.

Victoria Hampshire, VMD

Advanced Veterinary Applications, Bethesda, MD.

Paul Houghton

CE0, Biologist, Primate Products, Redwood City, CA.

Robert F. Hoyt, Jr., DVM, MS, DACLAM

Chief, Laboratory Animal Medicine and Surgery,

National Heart Lung and Blood Institute, NIH, Bethesda, MD.

Mary Lou James, BA, RLATG

Consultant, Regulatory Compliance, St. Louis, MO.

Alicia Z. Karas, DVM, MS, DACVA

Assistant Professor, Anesthesia, Department of Clinical Sciences,

Cummings School of Veterinary Medicine, Tufts University, North Grafton, MA.

Bruce W. Kennedy, MS, RLATG

Compliance Associate, Research and Sponsored Programs,

Cal Poly Pomona, Pomona, CA.

C. Max Lang, DVM, DACLAM

Professor and Chairman, Department of Comparative Medicine,

Milton S. Hershey Medical Center, Pennsylvania State University, Hershey, PA.

Richard H. Latt, DVM, DACLAM

Director, Animal Facilities, The Trudeau Institute, Saranac, NY.

Sherry M. Lewis, PhD

Nutritionist/Research Scientist, National Center for Toxicological Research,

Jefferson, AR

Carol Cutler Linder, PhD

Assistant Professor of Biology, New Mexico Highlands University, Las Vegas, NM.

John A. Maher, MS, MBA, CMAR, RLATG

Senior Manager, BioResources, Wyeth Research, Pearl River, NY.

Jörg Mayer, Dr.Med.Vet., MSc

Clinical Assistant Professor, Head of Exotics Service, Cummings School

of Veterinary Medicine, Tufts University, North Grafton, MA.

Fred W. Quimby, VMD, PhD, DACLAM

Director, Lab Animal Research Center, Rockefeller University, New York, NY.

John Curtis Seely, DVM, DACVP

Veterinary Pathologist, Expe
Research Triangle Park, NC.

esearch Triangle Park,

Director, Research Compliance, Ohio University, Athens, $\mathrm{OH}$.

Jerald Silverman, DVM, DACLAM

Professor and Director, Department of Animal Medicine,

University of Massachusetts Medical School, Worcester, MA.

Michael K. Stoskopf, DVM, PhD, DACZM

Mrofessor and Director of Environmental Medicine Consortium

College of Veterinary Medicine, North Carolina State University, Raleigh, NC.

Debra Tiano, MA, RLATG

Debra Tiano, MA, RLATG
Associate Director, Vivarium Operations, Emisphere Technologies, Tarrytown, NY.

Robert H. Weichbrod, PhD, MBA, RLATG

Animal Program Administrator, National Eye Institute, NIH, Bethesda, MD.

Axel Wolff, MS, DVM

Director, Division of Compliance Oversight, OLAW, NIH, Bethesda, MD.

\section{Refined intrathymic injection}

Those involved in animal research should continuously strive to minimize pain and distress by refining laboratory protocols. The refinement of surgical procedures, in particular, has great potential to improve conditions for laboratory animals-but investigators must be careful not to compromise efficacy, which can lead to the use of more animals or other problems. Here, de la Cueva et al. describe a new approach to direct intrathymic injection in mice, a procedure used in many experimental protocols. As currently constituted, this procedure requires exposure of the thoracic cavity, which can result in poor recovery and postsurgical complications for the mouse. Using their refined, minimally invasive technique, the authors show how accurate intrathymic injections can be given in such a way as to reduce pain and even eliminate the need for postoperative analgesia. See page 27

\section{Another alternative to rabbit intubation}

The distinct oropharyngeal anatomy of the rabbit makes airway management in these animals difficult. To improve surgical airway management of rabbits, researchers are searching for alternatives to endotracheal intubation, often adapting techniques used in humans and other mammals. Yamamoto et al. report their efforts to use the laryngeal tube to ventilate rabbits under surgical conditions. They tested the laryngeal tube in six healthy male New Zealand White rabbits and found that the device is a viable alternative to endotracheal intubation for airway management in rabbits. See page 33

\section{Helicobacter meets its match}

Health maintenance of rodent colonies requires constant vigilance on the part of animal caretakers at a time when the exchange of animals between laboratories and across borders is increasingly commonplace. Subclinical infections, such as those involving Helicobacter, are especially pernicious because they can compromise research without symptoms or fanfare. Moreover, once an infection is detected, it is difficult to eradicate and may necessitate costly and time-consuming rederivation of valuable transgenic and other rodents. Kostomitsopoulos et al. recount their recent experience with Helicobacter infection in a colony of newly arrived trangenic mice. Although the imported mice were valuable to the facility, rederviation was not an option. Instead, they used a four-antibiotic medicated feed to eliminate the Helicobacter over eight weeks. Subsequent retesting of the animals demonstrated that they remained Helicobacter free for more than 12 months. See page 37 